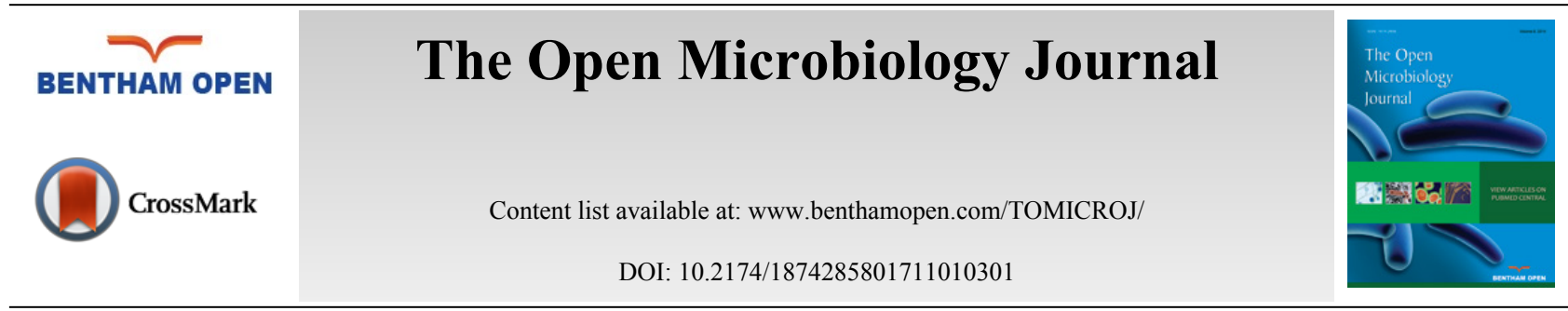

CORRIGENDUM

\title{
In Silico Design of a Chimeric Protein Containing Antigenic Fragments of Helicobacter Pylori; A Bioinformatic Approach
}

\section{Amir Ghasemi*}

Department of Microbiology and Immunology, Faculty of Medicine, Kashan University of Medical Sciences, Kashan, Iran

\section{REVISED VERSION NOVEMBER 2017}

The following list provides a description of the changes made to the publication since the original version of the article entitled "In Silico Design of a Chimeric Protein Containing Antigenic Fragments of Helicobacter pylori; A Bioinformatic Approach" was published online in May 2016.

\section{PAGE 97:}

In the Abstract, the Following Text Appears:

"In order to obtain such benefit in $\mathrm{H}$. pylori vaccine study, a chimeric gene containing four fragments of FliD sequence (1-600 bp), UreB (327-334 bp),VacA (744-805 bp) and CagL(51-100 bp) which have a high density of B- and T-cell epitopes was designed."

\section{This Should be:}

"In order to obtain such benefit in H. pylori vaccine study, a chimeric gene containing four fragments of FliD sequence (1-600 bp), UreB (327-385 bp),VacA (744-805 bp) and CagL(51-100 bp) which have a high density of B- and T-cell epitopes was designed."

\section{PAGE 98:}

In the paragraph 4, the following text appears:

"Urease B has been widely investigated as a potential antigen for the development of prophylactic and therapeutic vaccines against $\mathrm{H}$. pylori infection [32,33]. UreB(327-334) is considered as a good B cell epitope and has been found to be protective in mice $[34,35]$ "

\section{This Should be:}

"Urease B has been widely investigated as a potential antigen for the development of prophylactic and therapeutic vaccines against $H$. pylori infection [32,33]. UreB (327-385) is considered as a good B cell epitope and has been found to be protective in mice $[34,35]$."

\footnotetext{
* Address correspondence to this author at Department of Microbiology and Immunology, Faculty of Medicine, Kashan University of Medical Sciences, Kashan, Iran; Tel: 00989123595610; E-mail: ghasemia77@yahoo.com
} 


\section{PAGE 100:}

\section{In the Results, the Following Text Appears:}

"The nominated sequences for designing of chimeric construct were FliD (1-600), UreB (327-334), VacA (744-805) and CagL (51-100)."

\section{This Should be:}

“The nominated sequences for designing of chimeric construct were FliD (1-600), UreB (327-385), VacA (744-805) and CagL (51-100)."

\section{PAGE 102:}

\section{In the Paragraph 3, the Following Text Appears:}

"The secondary structure of the chimeric protein was predicted by several online programs, and the best result was achieved by GOR-IV as shown in Fig. (4). Results indicated total residues of 785 which were made up 140 strands (17.83\%), 299 helices (38.09\%) and 346 random coils (44.08\%). No predicted signal peptide was identified in the initial region of the protein sequence."

\section{This Should be:}

"The secondary structure of the chimeric protein was predicted by several online programs, and the best result was achieved by GOR-IV as shown in Fig. (4). Results indicated total residues of 790 which were made up 140 strands (17.72\%), 299 helices (37.85\%) and 351 random coils (44.43\%). No predicted signal peptide was identified in the initial region of the protein sequence."

\section{PAGE 107:}

\section{In the Discussion, the Following Text Appears:}

Based on our finding, a chimeric protein including immunodominant epitopes from different antigenic proteins such as FliD (1-600), UreB (327-334), VacA (744-805) and CagL (51-100) would likely induce strong comprehensive protective immunity.

\section{This Should be:}

Based on our finding, a chimeric protein including immunodominant epitopes from different antigenic proteins such as FliD (1-600), UreB (327-385), VacA (744-805) and CagL (51-100) would likely induce strong comprehensive protective immunity.

\section{PAGE 108:}

\section{In the Conclusion, the Following Text Appears:}

"Our data showed that the possibility of successful production of a large chimeric protein composing of four domains FliD (1-600), UreB(327-334), VacA (744-805) and CagL (51-100) in the prokaryotic host."

\section{This Should be:}

"Our data showed that the possibility of successful production of a large chimeric protein composing of four domains FliD (1-600), UreB(327-385), VacA (744-805) and CagL (51-100) in the prokaryotic host."

\section{(C) 2017 Amir Ghasemi.}

This is an open access article distributed under the terms of the Creative Commons Attribution 4.0 International Public License (CC-BY 4.0), a copy of which is available at: https://creativecommons.org/licenses/by/4.0/legalcode. This license permits unrestricted use, distribution, and reproduction in any medium, provided the original author and source are credited. 